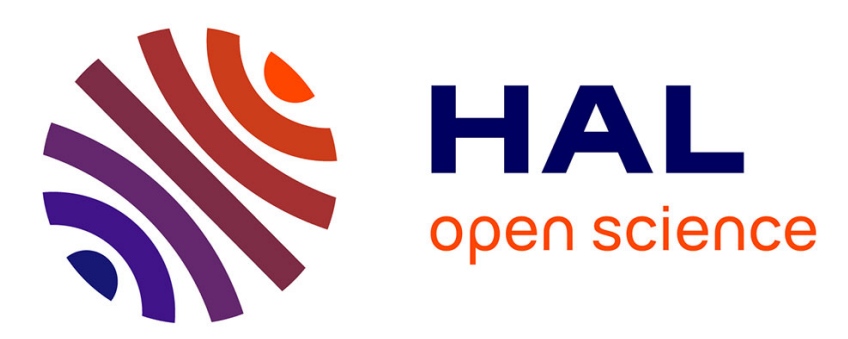

\title{
Réflexions sur l'identité individuelle et lignagère dans la noblesse du Dga' ldan pho brang à partir de l'étude des pratiques d'adoption et de mariage en gendre (1860-1959)
}

Alice Travers

\section{To cite this version:}

Alice Travers. Réflexions sur l'identité individuelle et lignagère dans la noblesse du Dga' ldan pho brang à partir de l'étude des pratiques d'adoption et de mariage en gendre (1860-1959). Hypotheses, 2007, 2007/1, pp.203-214. 10.3917/hyp.061.0203 . halshs-00688831

\section{HAL Id: halshs-00688831 \\ https://shs.hal.science/halshs-00688831}

Submitted on 3 Nov 2020

HAL is a multi-disciplinary open access archive for the deposit and dissemination of scientific research documents, whether they are published or not. The documents may come from teaching and research institutions in France or abroad, or from public or private research centers.
L'archive ouverte pluridisciplinaire HAL, est destinée au dépôt et à la diffusion de documents scientifiques de niveau recherche, publiés ou non, émanant des établissements d'enseignement et de recherche français ou étrangers, des laboratoires publics ou privés. 


\section{RÉFLEXIONS SUR L'IDENTITÉ INDIVIDUELLE ET LIGNAGÈRE DANS LA NOBLESSE DU DGA' LDAN PHO BRANG À PARTIR DE L'ÉTUDE DES PRATIQUES D'ADOPTION ET DE MARIAGE EN GENDRE (1860-1959)}

\section{Alice Travers}

Éditions de la Sorbonne | «Hypothèses »

2007/1 10 | pages 203 à 214

ISSN $1298-6216$

ISBN 9782859445782

Article disponible en ligne à l'adresse :

https://www.cairn.info/revue-hypotheses-2007-1-page-203.htm

Distribution électronique Cairn.info pour Éditions de la Sorbonne.

(C) Éditions de la Sorbonne. Tous droits réservés pour tous pays.

La reproduction ou représentation de cet article, notamment par photocopie, n'est autorisée que dans les limites des conditions générales d'utilisation du site ou, le cas échéant, des conditions générales de la licence souscrite par votre établissement. Toute autre reproduction ou représentation, en tout ou partie, sous quelque forme et de quelque manière que ce soit, est interdite sauf accord préalable et écrit de l'éditeur, en dehors des cas prévus par la législation en vigueur en France. Il est précisé que son stockage dans une base de données est également interdit. 


\section{Réflexions sur l'identité individuelle et lignagère dans la noblesse du Dga' ldan pho brang à partir de l'étude des pratiques d'adoption et de mariage en gendre (1860-1959)}

\section{Alice TRAVERS *}

Aux XIX et $\mathrm{XX}^{\mathrm{e}}$ siècles, la noblesse (tib. sku $\mathrm{drag}^{1}$; litt. " corps excellent ») du Dga' ldan pho brang, environ deux cent cinquante familles liées au gouvernement central de Lha sa, au Tibet, sous l'autorité du Dalaïlama, est avant tout une élite administrative héréditaire: ses membres se transmettent des domaines, dont la possession est liée à l'exercice obligatoire d'une charge au sein du gouvernement ${ }^{2}$. Les fonctionnaires (tib. Zhung zhabs) du gouvernement tibétain sont divisés en une branche monastique, dont le recrutement se fait dans toutes les couches de la société, et une branche laïque, au recrutement presque exclusivement nobiliaire. Les familles nobles laïques ont donc en commun le service du gouvernement et la possession de la terre. Mais la disparité entre les lignages (tib. mi rgyud) est

\footnotetext{
* Cette recherche s'inscrit dans le cadre plus large d'une étude prosopographique de la noblesse tibétaine, pour une thèse en cours sous la direction de Jean Duma (Paris X) et Heather Stoddard (Inalco), intitulée La noblesse du Tibet central de 1895 à 1959: essai de définition d'un groupe social par l'étude des carrières et des stratégies matrimoniales et lignagères.

1. J'utilise le système de translittération décrit dans T.V. WYLIE, «A Standard System of Tibetan Transcription ", Harvard Journal of Asiatic Studies, 22 (1959), p. 261-67.

2. Deux autres groupes de nobles sont présents à cette époque au Tibet central : l'un est lié au gouvernement de Bkra shis lhun po, sous l'autorité du Pan chen bla ma, l'autre au gouvernement de Sa skya sous l'autorité du Khri chen. Cependant, le groupe de nobles du Dga' ldan pho brang est le plus important en nombre ainsi qu'en termes de pouvoir et de prestige. Étant le mieux représenté dans les sources, il constitue mon objet principal d'étude.
} 
immense et ils sont hiérarchisés en plusieurs groupes ${ }^{3}$. Au sommet de la hiérarchie, les sde dpon, quatre familles issues des anciens rois et ministres de l'Empire tibétain (VII -IX siècle); les yab gzhis, six familles anoblies des précédents Dalaï-lamas; les mi drag, environ dix-huit familles riches et politiquement influentes; et enfin les autres familles de propriétaires terriens (tib. sger pa) qui jouissent aussi du privilège, ou de l'obligation, de servir le gouvernement.

L'identité de la noblesse tibétaine, principalement celle des chefs de maison ou héritiers, est basée sur la détention d'une charge mais aussi sur l'appartenance à un lignage 5 . Or cette appartenance est l'objet de variations liées à des contingences extérieures, parmi lesquelles la nécessité pour les lignages d'assurer leur pérennité en palliant l'absence d'héritiers par l'adoption ou par la pratique du mariage en gendre, un mariage uxorilocal où le gendre (tib. mag $p a$ ) prend le nom de la maison de sa femme et en devient l'héritier ou le cohéritier. Ces pratiques ne sont spécifiques ni au groupe nobiliaire ni à la période considérée. Il m'a pourtant semblé intéressant de m'interroger sur la nature des lignages au sein d'un groupe dont l'identité, davantage que dans le reste de la société tibétaine, repose sur la mémoire de ces lignages comme sur l'impérieuse nécessité de leur continuité. En effet, l'absence d'héritiers susceptibles de remplir le service gouvernemental implique immanquablement le retour à l'état de roture.

Les sources qui ont permis la reconstitution des généalogies et des histoires familiales de la noblesse tibétaine sont, d'une part, les informations disponibles dans les ouvrages de Luciano Petech et du prince Pierre de Grèce ${ }^{6}$, et, d'autre part, des sources primaires, écrites et orales. Les sources écrites sont constituées principalement des archives britanniques de l'India Office et du Foreign Office à Londres et à Delhi, et des biographies et autobiographies de nobles tibétains publiées au Tibet et en exil depuis 1970.

3. Le lignage est un "groupe de filiation moins étendu que le clan et souvent nommé dont les membres se considèrent comme descendants d'un ancêtre commun et sont effectivement à même de retracer généalogiquement ces relations (contrairement aux membres d'un clan)", cf. "Glossaire de la parenté », L'Homme, 154-155 (2000), p. 727. Le lignage est entendu comme formant une sorte de personne morale, un corps dont les membres ont en commun des droits et des devoirs. J'utiliserai le terme " lignée " pour désigner une simple succession d'individus.

4. J'emploierai ici le terme "famille" dans le sens suivant : "ensemble des personnes unies par le sang et par l'alliance, vivant au même pot et au même feu ", cf. ibid., p. 725 .

5. Entretien avec Glang mthong zur pa Rig bsam, né en 1941, 26/10/2003, Lhasa, RAT (Région autonome du Tibet).

6. L. PeteCH, Aristocracy and Government in Tibet, 1728-1959, Rome, 1973 (Serie Orientale Roma, XLV) ; P.P. OF GREECE AND DENMARK, A Study of Polyandry, La Haye, 1963. 
Les sources orales proviennent d'entretiens réalisés avec des nobles tibétains nés au début du XXe siècle. Cette étude portera donc sur les héritiers nobles recrutés en dehors de la lignée biologique, soit par adoption, soit par la pratique du mariage en gendre entre 1860 et $1959^{\circ}$.

Le passage des individus nobles d'un lignage à l'autre permet d'envisager l'identité sous son vrai jour : en tant que processus, construction et non en tant que permanence ${ }^{8}$; et dans ses deux dimensions, individuelle et collective, à travers l'étude des individus, des lignages et des relations des uns aux autres. Que nous apprennent ces pratiques d'adoption sur la nature du lignage et sur le statut de l'individu dans la noblesse tibétaine aux XIX et $\mathrm{XX}^{\mathrm{e}}$ siècles? Après une brève présentation du contexte de parenté et d'alliance dans la société tibétaine, et des pratiques d'adoption et de mariage en gendre, je tenterai d'éclairer la nature ambiguë de l'identité lignagère dans la noblesse tibétaine. Je m'interrogerai enfin sur les stratégies identitaires développées par les individus nobles dans le cadre de ces pratiques.

\section{Mariage en gendre et adoption, fréquence et modalités du phénomène dans la noblesse tibétaine}

La société tibétaine à l'époque étudiée est ce que l'on appelle une " société à maisons ", où l'importance de la maison prime sur la parentèle", la notion de résidence sur celle de filiation ${ }^{10}$. C'est autour de la maison comme édifice ou comme "ensemble de ceux qui habitent ensemble" que s'articulent toutes les relations sociales. Le nom que l'on porte est celui de la maison, acquis soit par la naissance, soit par le mariage, soit par l'adoption ${ }^{11}$.

7. J'ai relevé et étudié cinquante-cinq cas dont six d'adoptions, quarante-huit de mariages en gendre et un d'adoption suivie de mariage en gendre. Sauf dans le lignage Bshad sgra décrit plus loin, les individus retenus dans cette étude sont nés au plus tôt en 1860 ; 1959 correspond à la fin de l'organisation sociale et du gouvernement traditionnels du Tibet.

8. F-J RugGIU, "Les notions d'“identité", d'“individu” et de "self" et leur utilisation en histoire sociale" dans Identités, appartenances, et revendications identitaires, M. BELLISA, A. Bellavitis, M. CotTret, L. CroQ et J. Duma éd., Paris, 2005, p. 396.

9. Définition de la "maison " dans C. LÉVI-STRAUSS, "Histoire et ethnologie ", Annales ÉSC, 38-6 (1983), p. 1224 ; ou P. LAMAISON, "La notion de maison : entretien avec C. Lévi-Strauss ", Terrain : habiter la maison, 9 (1987), p. 34.

10. R.A. STEIN, La Civilisation tibétaine, Paris, [1962] 1981, p. 427 ; et B.N. AzIZ, Tibetan Frontier Families, Reflections of Three Generations from D'ing-ri, New Delhi, 1978 , p. 117.

11. B.N. AzIZ, op. cit., p. 27. La primauté du nom de la maison sur celui du père est flagrante dans certains cas : une femme, par exemple, se marie dans une autre maison. Elle donne naissance à un enfant, puis devient veuve et revient dans sa maison originelle, dont l'enfant prend alors le nom. Voir D.Y. YUTHOK, The House of the Turquoise Roof, New York, [1990] 1995. 
Dans des contextes similaires, on parle encore de "famille souche ${ }^{12}$ ". Plusieurs frères mariés peuvent rester ensemble au foyer sans diviser le patrimoine, formant ce que l'on appelle des "frérèches" " à la différence près qu'au Tibet ils sont parfois tous liés dans un seul mariage, selon le principe monomarital décrit par Melvyn Goldstein ${ }^{14}$. Il résulte de l'organisation de cette société que le lignage est basé sur la transmission du patrimoine, et donc sur la maison, et qu'il peut ainsi être tour à tour patrilinéaire ou matrilinéaire ${ }^{15}$, dans le cas où la mère est une héritière ayant fait venir un gendre, situation que l'on rencontre à d'autres époques et dans d'autres contextes, notamment européens, nobiliaires ou non ${ }^{16}$. En pays tibétain, l'existence de ces "lignages patrimoniaux » implique une grande diversité d'arrangements matrimoniaux ${ }^{17}$.

Comme dans toutes les sociétés à maison, le mariage en gendre et l'adoption marquent fortement le paysage familial de la noblesse tibétaine. Il est difficile de se faire une idée de l'importance relative de ces pratiques. Cependant, dans le groupe des quarante-trois familles nobles décrites par Luciano Petech et existant encore dans la deuxième partie du XIX et dans la première moitié du $\mathrm{XX}^{e}$ siècle ${ }^{18}, 42 \%$ des familles ont recours dans cette période au moins une fois au mariage en gendre ou à l'adoption ${ }^{19}$. On peut légitimement penser que la proportion réelle était bien supérieure, puisque pour les $58 \%$ des familles restants, l'auteur manquait le plus souvent d'information concernant les mariages.

12. Définition dans M. NASSIET, Parenté, noblesse et États dynastique, $X V^{f}-X V I$ siècle, Paris, 2000, p. 364.

13. J. GOODY, Famille et mariage en Eurasie, Paris, 2000, p. 145 et 151.

14. M.C. GoldSTEIN, "Stratification, Polyandry, and Family Structure in Central Tibet ", Southwestern Journal of Anthropology, 27-1(1971), p. 71.

15. La définition du lignage tibétain differe en cela du sens que l'on donne habituellement en sciences sociales à ce terme, à savoir un groupe de filiation unilinéaire (du côté du père ou bien de la mère) ou indifférencié (qui englobe les deux côtés de façon indifférenciée).

16. C. LÉVI-STRAUSS, art. cité, p. 1225 ; É. ClaVERIE et P. LAMAISON, L'Impossible mariage. Violence et parenté en Gévaudan, $17,18^{e}$ et $19^{\circ}$ siècles, Paris, 1982, p. 105.

17. Cf. définition de la «lignée patrimoniale ": " ensemble formé des héritiers successifs du bien détenu par le couple souche", ibid., p. 360. On retrouve dans la noblesse du Tibet central toutes les formes traditionnelles du mariage tibétain, à savoir la monogamie, la polyandrie unigénérationnelle, fraternelle ou non, et bigénérationnelle, la polygynie, sororale ou non.

18. Son livre concerne les quarante-sept familles les plus influentes sur le plan politique entre 1728 et 1959.

19. Quinze familles ont eu recours au mariage en gendre (dont quatre familles à deux reprises), deux familles à l'adoption (dont une par deux fois) et une famille aux deux à la fois. 
L'adoption est en général au Tibet une adoption simple ${ }^{20}$, qui laisse subsister des liens avec la famille d'origine, plutôt qu'une adoption plénière, où il $\mathrm{y}$ a rupture des liens avec la famille d'origine ${ }^{21}$. Elle est présente de façon récurrente dans la noblesse tibétaine au sein des lignages de fonctionnaires ecclésiastiques, où les héritiers sont des moines et ne peuvent produire de successeur biologique. Ils ont donc recours presque systématiquement à l'adoption d'un héritier, qui se fait en général dans la proche parenté. C'est le cas notamment du lignage noble de fonctionnaires ecclésiastiques Bkras khang ${ }^{22}$. Les lignages de fonctionnaires laïcs ont également recours à cette pratique $e^{23}$, par exemple dans la maison Lha klu ou Lha sdings ${ }^{24}$. En général l'adoption n'intervient qu'en l'absence de fille, à cause de la fréquence et du caractère banal du mariage en gendre.

Les sources et la littérature secondaire légitiment d'étudier comme un seul et même objet l'adoption et le mariage en gendre. En effet, le mariage en gendre y est très souvent assimilé à une adoption ${ }^{25}$. Du reste, les anthropologues anglais n'hésitent pas à employer le terme adoptive bridegroom pour désigner les individus qui contractent ce type d'union. D'un autre côté, l'adoption est très souvent, comme l'a montré Suzanne Lallemand, un acte d'alliance ${ }^{26}$. Dans mon corpus, les adoptions et les mariages en gendre s'inscrivent, au même titre que les autres types d'alliances, dans les cycles d'échanges entre lignages. Enfin, dans la noblesse tibétaine, une personne adoptée se marie souvent dans la parenté proche de

20. Différents termes comportant diverses nuances comme bu skal, bu tshab ou bu dod permettent de désigner un individu adopté en tibétain.

21. Elle se distingue bien du fosterage, cession momentanée de la progéniture, qui existe également au Tibet mais que je n'ai pas retenue dans cette étude. La distinction entre fosterage et adoption a été inaugurée par J. GOODY, "Adoption in Cross-cultural Perspective ", Comparative Studies in Society and History, 11-1 (1969), p. 55-78; voir, Adoption et fosterage, M. CORBIER dir., Paris, 1999.

22. Entretien avec Bkras khang mkhan chung, né en 1918, 05/09/2003, Dharamsala, Inde, et L. PETECH, op. cit., p. 109.

23. P.P. OF GREECE AND DENMARK, op. cit., p. 545.

24. Pour la maison Lha klu, cf. Who's Who in Tibet, Government of India Press, Calcutta, India, 1949, p. 64, India Office Records (=IOR)/L/P\&S/20/D220/2, Londres, et L. PETECH, op. cit., p. 48 ; pour la maison Lha sdings, cf. List of Chiefs and Leading Families in Sikkim, Bhutan and Tibet, Government of India Press, Calcutta, India, 1933, p. 16, IOR/L/P\&S/20/D216, Londres, et L. PETECH, op. cit., p. 196.

25. Dans les sources par exemple, pour parler de Zur khang Bsod nams dbang 'dus et de son mariage en gendre : "Is a brother of Surkhang I and was adopted into the family of Kunsangtse ", Who's Who in Tibet, 1949, op. cit., p. 54 ; ou dans certains entretiens, cf. Tsha rong Bdud 'dul rnam rgyal, né en 1920, 04/09/2004, Kalimpong, Inde. En ce qui concerne la littérature secondaire, op. cit., M. CORBIER dir., p. 20 ; et S. Lallemand, La Circulation des enfants en société traditionnelle, Prêt, don, échange, Paris, 1993, p. 60.

26. Ibid. 
sa famille d'adoption ${ }^{27}$. On assiste en fait à un phénomène observé par Claude Lévi-Strauss dans toutes les sociétés à maison, un brouillage des catégories de l'alliance et de la filiation ${ }^{28}$.

L'adoption et le mariage en gendre ont une visée successorale ${ }^{29}$, aussi les maisons ont tendance à les redoubler : la maison Zhol khang prend ainsi deux mag pa de la maison Mdo zur pour ses deux filles cadettes; chez les Glang mthong, le chef de maison se retrouvant sans héritier décide d'adopter deux des fils de son frère cadet qui avait été marié en gendre auparavant dans la maison Zhol smad ${ }^{30}$. Le même objectif, celui d'assurer au mieux sa succession, explique que l'on fasse parfois venir un gendre, alors que l'on dispose déjà d'un héritier de sang dans la famille. Dans ce cas, l'héritier de sang assumera le service gouvernemental tandis que le mag pa venu de l'extérieur prendra le rôle de gzhis bzhugs (litt. " celui qui reste sur le domaine "), c'est-à-dire d'administrateur du domaine. C'est donc dans le contexte d'une forte mobilité des individus et d'une singulière diversité de solutions apportées au problème de la transmission du patrimoine que la question de l'identité des lignages et des individus se pose.

\section{Sang ou maison? Les tensions à l'œuvre dans l'identité lignagère de la noblesse tibétaine}

Il est couramment admis, à propos des sociétés à maison en général ${ }^{31}$, et de la noblesse tibétaine en particulier, que le sang ou le biologique ne joue qu'un très faible rôle dans le fonctionnement et la représentation des lignages $^{32}$. Pourtant, dans leurs discours, les nobles tibétains considèrent que la lignée biologique est un idéal. Il est intéressant de noter que plusieurs nobles interrogés à ce sujet prétendent que la pratique de l'adoption et du mariage en gendre pour le recrutement des héritiers n'est qu'une exception, alors que nous avons vu à quel point elle était fréquente ${ }^{33}$. Par ailleurs, les nobles tibétains tentent réellement de privilégier les liens de sang dans le choix des héritiers et adoptent de préférence un neveu ou tout autre individu

27. Barbara Aziz a observé ce phénomène chez les Tibétains de Ding ri, cf. B.N. AzIZ, op. cit., p. 124.

28. C. LÉVI-STRAUSS, art. cité, p. 1224.

29. Dans d'autres contextes d'adoption, le but poursuivi est celui de l'échange de services entre générations, voir op. cit., M. CORBIER dir., p. 35.

30. Famille de las drung ou de lettrés au service du gouvernement tibétain, qui avaient des domaines mais ne faisaient pas partie de la noblesse.

31. C. LÉVI-STRAUSS, art. cité, p. 1224.

32. T. SHEN et S. LIU, Tibet and the Tibetans, Stanford, 1953, p. 110 et 142 ; et L. PETECH, op. cit., p. 18.

33. Entretiens avec Rdo dgon pa Gsang bdag rdo rje, né en 1945, 24/11/2005 et 23/01/2006, et Brag gdong bkras gling Dbang rdor, né en 1934, 20/01/2006, Lhasa, RAT. 
lié par le sang. L'exemple de la famille Glang mthong peut à nouveau éclairer notre propos: les deux neveux adoptés par le chef de lignage appartiennent certes à une autre maison, mais présentent l'avantage d'être liés par le sang à la lignée Glang mthong ${ }^{34}$. Dans certaines maisons comme celle des Rdo dgon pa, les contrats de mariage précisaient, pour la glorifier, que leur lignée était ininterrompue (tib. mi rgyud ma chad pa), puisqu'ils n'avaient jamais eu recours au mariage en gendre ${ }^{35}$. Enfin, dans le cas de Zla bzang dgra'dul (1888-1959), roturier anobli par le XIII ${ }^{e}$ Dalaï-lama et marié en gendre, en 1913, à la veuve du futur héritier Tsha rong, il semblerait que l'idée de le marier de surcroît à trois sœurs du défunt visait à garantir que les futures générations aient du sang Tsha rong dans les veines ${ }^{36}$.

Pourtant, cette préférence affirmée pour les liens du sang ne joue qu'un rôle limité dans la pratique. Certes, ces arrangements ne résultent pas toujours d'un choix délibéré. Il peut arriver que l'héritier unique soit malade, ou faible d'esprit ${ }^{37}$. De même, la succession chronologique des événements, lorsqu'elle est connue, explique souvent la logique de la situation : il se peut que le fils sur lequel on avait compté décède subitement et que le mariage en gendre d'une fille cadette permette de rattraper bien à propos la situation; ou encore lorsque, on a fait venir un mag pa pour une fille aînée, c'est parfois parce qu'on a préféré assurer d'emblée la continuité du lignage avant d'avoir la certitude que les fils suivants atteindraient l'âge adulte.

Mais s'il importait vraiment que l'héritier soit du sang de la lignée biologique, on comprendrait mal que l'on fasse parfois venir un mag $p a$ alors que la famille comporte déjà des fils en âge d'hériter dont elle se sépare ${ }^{38}$. Certaines contraintes, notamment religieuses, sont reconnues comme valides et légitimement susceptibles de priver un père de la possibilité de faire de son fils un héritier : par exemple, si l'horoscope d'un fils indique que sa vie

34. Entretiens avec Glang mthong zur pa Tshe brtan sgrol ma, née en 1934, $10 / 09 / 2003$, et Glang mthong zur pa Blo bzang chos spel, né en 1939, 23/10/2005, Dharamsala, Inde; avec Glang mthong zur pa Yon tan rgya mtsho, né en 1943, $8 / 01 / 2006$, et Glang mthong Thub bstan lha mo, née en 1945, 10/01/2006, Lhasa, RAT. La maison Lha sdings pratique aussi l'adoption d'un neveu, cf. List of Chiefs and Leading Families in Sikkim, Bhutan and Tibet, 1933, op. cit., p. 16.

35. On note à ce propos que la même expression sert à parler d'une lignée ininterrompue, car sans mag pa, et d'une lignée ininterrompue grâce à la pratique du mariage en gendre. Entretiens avec Rdo dgon pa Gsang bdag rdo rje, né en 1945, $24 / 11 / 2005$ et $23 / 01 / 2006$.

36. R.D. TARING, Daughter of Tibet, Londres, [1970] 1986, p. 40.

37. Si c'était seulement le cas de l'aîné d'une fratrie, cela serait sans importance car, chez les Tibétains comme en Gévaudan, on "fait l'aîné ", le principe de primogéniture est non pertinent, P.P. OF GREECE AND DENMARK, op. cit., p. 419.

38. Phénomène observé par T. SHEN and S. LIU, op. cit., p. 142. 
sera courte ou qu'il est préférable qu'il devienne moine, ou encore s'il est reconnu comme la réincarnation d'un maître religieux et qu'il doit quitter la maison pour rentrer au monastère.

De plus, il n'est pas du tout exceptionnel que l'on dispose de fils bien portants mais que l'on commence par les donner à d'autres familles, sans doute pour répondre à des obligations d'échanges matrimoniaux. Ainsi, dans le lignage Zur khang, dans la deuxième moitié du XIX siècle, deux fils aînés sont donnés en mag pa à deux autres familles, les Bkras gshongs et les Bshad sgra, et l'on fait venir un gendre de la famille Lcang lo can pour épouser Tshe brtan g.yu gron, une fille de la maison, et devenir l'héritier Zur khang ${ }^{39}$.

L'exemple des tentatives désespérées de la famille Bkras gshongs pour s'assurer un héritier est tout à fait parlant ${ }^{40}$, bien qu'il représente un cas extrême. Cette famille sans enfants ne réussit pas à adopter un fils de la maison Zur khang à laquelle ils étaient déjà liés par alliance et qui leur avait été promis. En 1929, les Bkras gshongs décident alors d'adopter une fille Zur khang, Rdo rje g.yu gron, dans l'idée qu'elle prendrait par la suite un mag pa. Il est intéressant de noter qu'à la génération suivante, le nouvel héritier de la famille Bkras gshongs aurait été le fils de deux membres de deux autres maisons, ce qui confirme que la résidence prime bien sur le biologique. La cérémonie d'adoption, note l'intéressée, ressemble fort à un mariage, puisque la fille quitte définitivement la maison, est parée de la même façon qu'une mariée et reçoit une dot et une servante personnelle de sa maison d'origine. Mais, après quelques années sur place, elle est demandée en mariage par un héritier qui refuse de devenir mag pa et elle quitte sa maison d'adoption. C'est alors que l'on envisage une autre solution : on envoie Lha dbang sgrol ma, petite sœur de Rdo rje g.yu gron, pour qu'elle devienne la nouvelle héritière de la famille Bkras gshongs. Elle épouse un héritier d'une autre maison, Shel dkar gling pa, qui accepte de devenir mag pa. Ils mélangent pour un temps leurs deux lignages, comptant les séparer à nouveau à la génération suivante entre leurs enfants. Mais les deux époux se disputent et portent l'affaire devant le régent (tib. sde srid) du moment, qui décide finalement de confisquer toutes les propriétés de la famille Bkras gshongs : cette maison disparaît pour de bon ${ }^{41}$. On voit à quel point les héritiers potentiels adoptés par cette famille sont interchangeables,

39. Tshe brtan g.yu gron avait été mariée auparavant dans la famille Khri smon mais, après le décès de son mari, elle était revenue chez elle, voir D. Y. YUTHOK, op. cit., p. 32 et 314. Dans la famille Thon pa également, un fils est donné à une autre famille comme mag pa tandis qu'un fils Bde skyid gling est marié en gendre et devient l'hériter Thon pa, L. PETECH, op. cit., p. 69.

40. Ibid., p. 105-108.

41. Ibid., p. 131-133. 
ce qui tend à montrer combien l'identité individuelle est reléguée au second plan par rapport à l'identité lignagère.

On observe finalement une tension entre un impossible idéal, celui de la continuité biologique, et le poids des contraintes naturelles (une très forte mortalité), religieuses (la reconnaissance des réincarnations ou les prédictions des horoscopes) ou sociales (les obligations matrimoniales envers d'autres maisons qui peuvent obliger à se séparer d'un héritier pour le donner à un autre lignage). Cette tension est à la base de l'identité lignagère dans la noblesse tibétaine.

\section{La double appartenance des héritiers adoptés, stratégies identitaires individuelles et lignagères}

Certes, dans le champ des alliances matrimoniales et dans la vie sociale, le noble tibétain, en particulier l'héritier, n'est que le représentant de son lignage : on ne se réfere à lui que par le nom de sa maison ou de son domaine héréditaire et l'on ne connaît que rarement son prénom ${ }^{42}$. Que se passe-t-il donc dans le cas des adoptions et des mariages en gendre, où l'héritier passe d'un lignage à l'autre? J'analyserai la place des individus au sein du lignage d'adoption et la façon dont ils investissent et utilisent cette double appartenance lignagère.

Pour percevoir les éventuels changements d'identité des nobles, plusieurs objets d'étude se présentent à nous, parmi lesquels les noms, les titres ou appellations d'honneur, et les carrières. En premier lieu, l'analyse des carrières tend à montrer que les héritiers adoptés étaient pleinement intégrés dans leur maison d'adoption et n'étaient pas désavantagés par le fait qu'ils étaient des "pièces rapportées " ${ }^{43}$, notamment lorsqu'ils provenaient de familles de statut inférieur. L'examen des carrières de la maison Bshad sgra, l'une des plus puissantes familles de mi drag, qui a eu très

42. La liste (tib. mtshan tho) de fonctionnaires laïcs établie en 1924 par le gouvernement tibétain et reproduite par Luciano Petech, utilise pour différencier les individus les distinctions suivantes: "fils" (tib. $b u$ et sras, à l'honorifique pour les familles de l'aristocratie) et "le jeune"(tib. gzhon pa), cf. L. PETECH, op. cit., p. 240-249. Dans leurs Who's Who, les Britanniques attribuaient des numéros aux individus d'une même famille.

43. On considère en général que le statut de mag pa est fort déprécié dans la société tibétaine, voir P.P. OF GREECE AND DENMARK, op. cit., p. 542. En Chine, le gendre adopté est généralement méprisé, cf. F. LAUWAERT, "Les fils du temps. À propos de l'adoption chinoise " dans op. cit., M. CORBIER dir., p. 90. Je n'ai rien rencontré de tel dans la noblesse tibétaine, où les mariages en gendre permettent à des cadets de devenir héritiers et de faire de belles carrières, comme l'avaient déjà noté T. SHEN and S. LIU, op. cit., p. 142. 
souvent recours à l'adoption et au mariage en gendre ${ }^{44}$, est très révélateur. Les mag pa n'y ont pas fait des carrières moins brillantes que les héritiers de sang, bien au contraire. Si l'on se base sur la description qu'en fait Luciano Petech, entre la deuxième moitié du XVIII siècle et jusqu'en 1959, sur sept générations et dix héritiers ${ }^{45}$, on trouve trois héritiers magpa et deux héritiers adoptés, mais toujours dans une branche du lignage Bshad sgra. Sur les sept héritiers de sang, on ne connaît la carrière détaillée que de cinq d'entre eux, parmi lesquels on compte trois ministres (tib. bka'blon), un individu décédé juste avant d'être nommé à ce poste, et enfin un dernier représentant du lignage, dont la carrière a été prématurément interrompue par la fin du gouvernement traditionnel tibétain en 1959. Ainsi, les héritiers Bshad sgra ont occupé de façon récurrente le prestigieux poste de ministre, qui confere le troisième rang. Parmi les trois héritiers venus de l'extérieur et mariés en gendre, l'un a fait une carrière légèrement inférieure, en devenant grand comptable (tib. rtsis dpon), un poste conférant le quatrième rang ${ }^{46}$, mais les deux autres ont occupé les postes les plus importants qui soient dans le gouvernement tibétain, les seuls à conférer le deuxième rang, à savoir le poste de régent pour Dbang phyug rgyal po ${ }^{47}$, et celui de Premier ministre (tib. blon chen) pour Dpal 'byor rdo rje. Or ces deux individus provenaient de familles de la petite noblesse, respectivement Spel bzhi et Shan kha ba. Le statut de mi drag de leur puissante maison adoptive Bshad sgra a sans doute été décisif, en plus de la reconnaissance de leurs capacités personnelles, pour les faire accéder à ces sommets politiques. En fait, le mariage en gendre et l'adoption se font, pour mon corpus et du point de vue des hommes ${ }^{48}$, dans $52 \%$ des cas, entre nobles de même rang hiérarchique ; dans $33 \%$ des cas, vers des lignages de rang supérieur; et seulement dans $15 \%$ des cas, vers des lignages de rang inférieur. Ils représentent donc souvent une opportunité d'ascension sociale, dans la mesure où, même dans les situations d'homogamie, des cadets se voient promus au statut d'héritier.

44. De 1864 à 1919, sur trois générations, pas un seul chef du lignage n'est de sang Bshad sgra: L. PETECH, op. cit., p. 17 et 180-184, et entretien avec Bshad sgra Dga' ldan dpal 'byor, né en 1923, 14/01/2006, Lhasa, RAT.

45. La mort prématurée des héritiers entraîne parfois la présence de plus d'un héritier par génération.

46. Les charges et titres du gouvernement tibétain étaient répartis selon un système de rang à sept degrés.

47. Plus haut poste du gouvernement après le Dalaï-lama et unique occurrence dans l'histoire du Tibet d'un laï l'occupant.

48. Les adoptions concernent majoritairement les hommes, l'exemple évoqué plus haut de la fille Zur khang étant unique. Sur les cinquante-cinq familles retenues, trentequatre cas d'homogamie, quatorze cas d'hypogamie (situation dans laquelle une femme est mariée à un homme de rang inférieur) et sept cas d'hypergamie. 
L'adoption et le mariage en gendre ont parfois un rôle tactique, celui d'opérer un changement complet d'identité, dans trois types de circonstances. Dans le premier type, il s'agit d'individus en mauvaise posture - par exemple lorsque le père a été destitué par le gouvernement et s'est vu confisquer tous ses biens - et qui se trouvent en conséquence interdits de participation au gouvernement. C'est le cas entre autres de Tshe dbang rdo rje, fils aîné de Lung shar, qui a été adopté en 1936 comme héritier de la famille Lha klu après que son père a été arrêté et emprisonné. Grâce à cette adoption, il peut non seulement retrouver une place d'héritier dans une puissante maison, mais aussi réaliser une très belle carrière de fonctionnaire $^{49}$. Autre exemple, au tournant du XX $\mathrm{X}^{\mathrm{e}}$ siècle, le fils de Rang zur, qui devient, juste après la dégradation de son père et la confiscation des domaines familiaux, mag pa dans la famille Skyid smad ${ }^{50}$. Le deuxième type de circonstances concerne les situations d'anoblissement, où l'adoption et le mariage en gendre vont permettre de mieux effacer une origine roturière ${ }^{51}$. Enfin, ces procédés permettent de donner une nouvelle identité légitime et de remettre dans le circuit professionnel les fils naturels des membres de la noblesse : ainsi, Phun tshogs rab rgyas (?-1920), fils illégitime de Lha klu lha lcam et Glang mdun gung, se fait adopter comme héritier de la maison Lha klu ${ }^{52}$; de même, Ngag dbang 'jigs med, fils illégitime né en 1909 d'une nonne, sœur de Hor khang dza sag, est marié en gendre dans la maison Nga phod et y fera, en tant qu'héritier, une très brillante carrière ${ }^{53}$.

Si les gendres et fils adoptifs bénéficient pleinement du statut de leur famille adoptive, ils n'en perdent pas pour autant les prérogatives de leur famille d'origine lorsque celle-ci est de rang supérieur. Il en est ainsi pour Tshe bdang don grub, né en 1907 dans la prestigieuse maison de yab gzhis Bsam grub pho brang, qui entre par mariage en gendre dans une famille noble de rang ordinaire, les Mtsho sgo, mais continue à se faire appeler par le titre sras (litt. "fils" à l'honorifique), auquel n'ont droit que les fils de grande noblesse, c'est-à-dire les sde dpon, yab gzhis et mi drag ${ }^{54}$. Il arrive enfin qu'un noble marié en gendre à une roturière parvienne à rester noble en

49. IOR/L/P\&S/12/4193, Londres, et Who's Who in Tibet, 1949, op. cit., p. 64.

50. Chiefs and Leading Families in Sikkim, Bhutan and Tibet, Calcutta, Superintendent Government Printing, India, 1915, p. 17, National Archives, FO/371/2318, Londres.

51. L'exemple du roturier anobli puis marié en gendre dans la maison Tsha rong a été examiné plus haut.

52. L. PETECH, op. cit., p. 48.

53. Who's Who in Tibet, 1949, op. cit., p. 82.

54. Ibid., p. 139. Cependant, il semblerait que les individus ne soient pas toujours maîtres du jeu : en effet, à l'inverse, lorsque Rig 'dzin rdo rje, issu de la famille noble de rang ordinaire Skyid sbug et marié en gendre dans la famille de mi drag Kun bzang rtse, rentre au gouvernement en 1945, celui-ci lui interdit de porter le titre de sras nang pa auquel sa nouvelle maison d'adoption lui donnait théoriquement droit, ibid., p. 59. 
anoblissant le lignage dont il prend le nom: dans les années 1920, Ka shod pa Mi'gyur rdo rje épouse Me togs, fille de la famille de serfs (tib. mi ser) 'Brang stod pa. Après un long procès qui oppose le couple à la famille noble Rdo ring, à laquelle appartenait Me togs et qui voulait que cette dernière continue à s'acquitter des taxes et corvées qu'elle leur devait, Mi 'gyur rdo rje obtient gain de cause et, conservant sa charge au gouvernement, commence un nouveau lignage noble ${ }^{55}$. Les individus adoptés restent donc liés à leurs familles d'origine tout en étant pleinement intégrés à leur famille d'adoption. En fonction de l'intérêt du moment, il leur arrive d'utiliser et de revendiquer l'une ou l'autre de leurs appartenances lignagères.

Ainsi, bien que le discours privilégie, comme dans d'autres noblesses, les liens de sang comme idéal pour la transmission lignagère, la fréquence et le contexte des pratiques d'adoption et de mariage en gendre dans la société tibétaine confirment que le biologique est relégué au second plan face à des relations plus fortes: d'une part, celle qui unit les lignages, avec les obligations d'échange qui en découlent; et d'autre part, l'appartenance au groupe noble tout entier. Les modalités de recrutement des héritiers montrent que, plus que l'appartenance au même sang, c'est le statut noble qui fait d'un individu l'héritier potentiel de tous les lignages nobles ${ }^{56}$.

On peut se demander si ce détachement de la transmission par le sang, observé dans la pratique, n'est pas en partie le fruit du contexte religieux dans lequel évolue la société tibétaine. En effet, la croyance en la réincarnation qui caractérise le bouddhisme, ainsi que l'existence de lignées de transmission religieuse, par réincarnation (tib. sku rgyud) ou de maitre à disciple (tib. slob rgyud), ne favoriseraient-elles pas dans toute la société, l'adhésion à ces lignées patrimoniales - le patrimoine pouvant être matériel ou spirituel - affranchies de leur base biologique?

55. Entretiens avec Ka shod pa 'Jam dbyangs chos rgyal, né en 1938, 13/03/2006, Londres, et Brag gdong bkras gling Dbang rdor, né en 1934, 20/01/2006, Lhasa, RAT. 56. Entretiens avec Rdo dgon pa Gsang bdag rdo rje, né en 1945, 24/11/2005 et 23/01/2006, et Brag gdong bkras gling Dbang rdor, né en 1934, 20/01/2006, Lhasa, RAT. 